\title{
Cortical dynamics of visual persistence and temporal integration
}

\author{
GREGORY FRANCIS \\ Purdue University, West Lafayette, Indiana
}

\begin{abstract}
In a temporal integration experiment, subjects must integrate two visual stimuli, presented at separate times, to perform an identification task. Many researchers have assumed that the persistence of the leading stimulus determines the ability to integrate the leading and trailing stimuli. However, recent studies of temporal integration have challenged that hypothesis by demonstrating that several theories of persistence are incompatible with data on temporal integration. This paper shows that an account of visual persistence given by a neural network model of preattentive vision, called the boundary contour system, explains data on temporal integration. Computer simulations of the model explain why temporal integration becomes more difficult when the display elements are separated by longer interstimulus intervals or are of longer duration or of higher luminance, or are spatially closer together. The model suggests that different mechanisms underlie the inverse duration effects for leading and for trailing elements. The model further predicts interactions of spatial separation, duration, and luminance of the trailing display.
\end{abstract}

Studies of temporal integration divide a visual stimulus pattern into small parts and distribute those parts across two images. Subjects view the images in rapid succession (possibly separated by an interstimulus interval, ISI) and perform a task that requires temporal integration of the displays. For example, Hogben and Di Lollo (1974) used a display consisting of a $5 \times 5$ matrix and two frames of 12 dots each. The subject's task was to report the location of the missing dot among the two frames. A key assumption of many studies is that temporal integration occurs when percepts generated by the leading stimulus persist and coexist with percepts generated by the trailing stimulus. Dixon and Di Lollo (1994) referred to this theory as the intrinsic persistence hypothesis. If this hypothesis is true, then stimulus characteristics affecting visual persistence should also affect temporal integration tasks. Many studies seem to support this hypothesis.

However, Dixon and Di Lollo (1994) argued against the intrinsic persistence hypothesis as an explanation of the properties of temporal integration. Studies of integration show that images are rarely retinotopically integrated across saccadic eye movements (Haber, 1983) and that blurring of moving stimuli is much less severe than measures of persistence predict (Burr, 1980). These results imply that persistence of isolated stimuli does not agree with properties of integration. Moreover, they argued that if integration is due to the persistence of the leading display's elements, properties of the trailing display's elements should not affect integration. They also replicated

The author extends his thanks to Vincent Di Lollo for helpful comments on an earlier version of this manuscript. The author's mailing address is Department of Psychological Sciences, 1364 Psychological Sciences Building, West Lafayette, IN 47907-1364 (e-mail: gfrancis@) psych.purdue.edu). findings from Groner, Bischof, and Di Lollo (1988), which showed that increasing the duration of the trailing display impaired performance substantially. According to Dixon and Di Lollo, these results suggest that the intrinsic persistence hypothesis cannot account for the properties of temporal integration without invoking additional mechanisms.

As an alternative to the intrinsic persistence hypothesis, Dixon and Di Lollo (1994) proposed the temporal coding hypothesis. They suggested that percepts of integration emerge from the way the visual system codes successive stimuli. Even if presented separately, stimuli can be coded as coextensive, implying that they are likely to be grouped together as parts of a single event. Likewise, when successive stimuli are coded as disjoint, they are likely to be perceived as parts of separate events. Dixon and Di Lollo (1994) described a mathematical model of the temporal coding hypothesis that calculates the correlation of signals from the leading and trailing displays of an integration study. Dixon and Di Lollo (1994) and Di Lollo, Hogben, and Dixon (1994) showed that the temporal correlation model can fit several data sets on temporal integration without invoking the intrinsic persistence hypothesis.

In distinction to those findings, this paper argues that within the framework of a neural network model of visual perception proposed by Grossberg and Mingolla (1985a, $1985 \mathrm{~b}$ ), the properties of visual persistence do explain characteristics of temporal integration. In this network, excitatory feedback loops generate reverberatory activations that persist beyond stimulus offset. Francis, Grossberg, \& Mingolla (1994) showed that inhibitory signals produced at stimulus offset or by successive stimuli reset the reverberations and explained several properties of visual persistence. New simulations show that application 
of the intrinsic persistence hypothesis allows the model to account for five known properties of temporal integration:

ISI: Increasing the duration of the ISI produces worse performance (Di Lollo et al., 1994).

Inverse duration with leading display: Increasing the duration of the leading display produces worse performance (Di Lollo et al., 1994; Dixon \& Di Lollo, 1994).

Inverse intensity: Increasing the luminance of the display elements produces worse performance (Di Lollo et al., 1994).

Inverse duration with trailing display: Increasing the duration of the trailing display produces worse performance (Dixon \& Di Lollo, 1994; Groner et al., 1988).

Inverse proximity: Performance worsens when the display elements are closer together (Di Lollo \& Hogben, 1987).

In addition, the model predicts two untested properties of temporal integration:

Inverse proximity and trailing display duration: The inverse duration effect should weaken as spatial separation increases.
Inverse proximity and trailing display intensity: The inverse intensity effect should weaken as spatial separation increases.

The data, predictions, and simulations are discussed in greater detail below. The next section describes the neural network model.

\section{RESONANCE AND VISUAL PERSISTENCE}

The current results use the boundary contour system (BCS) model of visual perception to explain properties of temporal integration. A complete account of this model and its relations to other parts of visual perception have been reviewed elsewhere (e.g., Grossberg, 1994) and is beyond the scope of this paper. The model is schematized in Figure 1. Briefly, pairs of like-oriented simple cells that are sensitive to opposite contrast polarities send their rectified output signals to like-oriented complex cells. Complex cells activate hypercomplex cells through on-center off-surround connections (first competitive stage), whose off surround carries out an end-

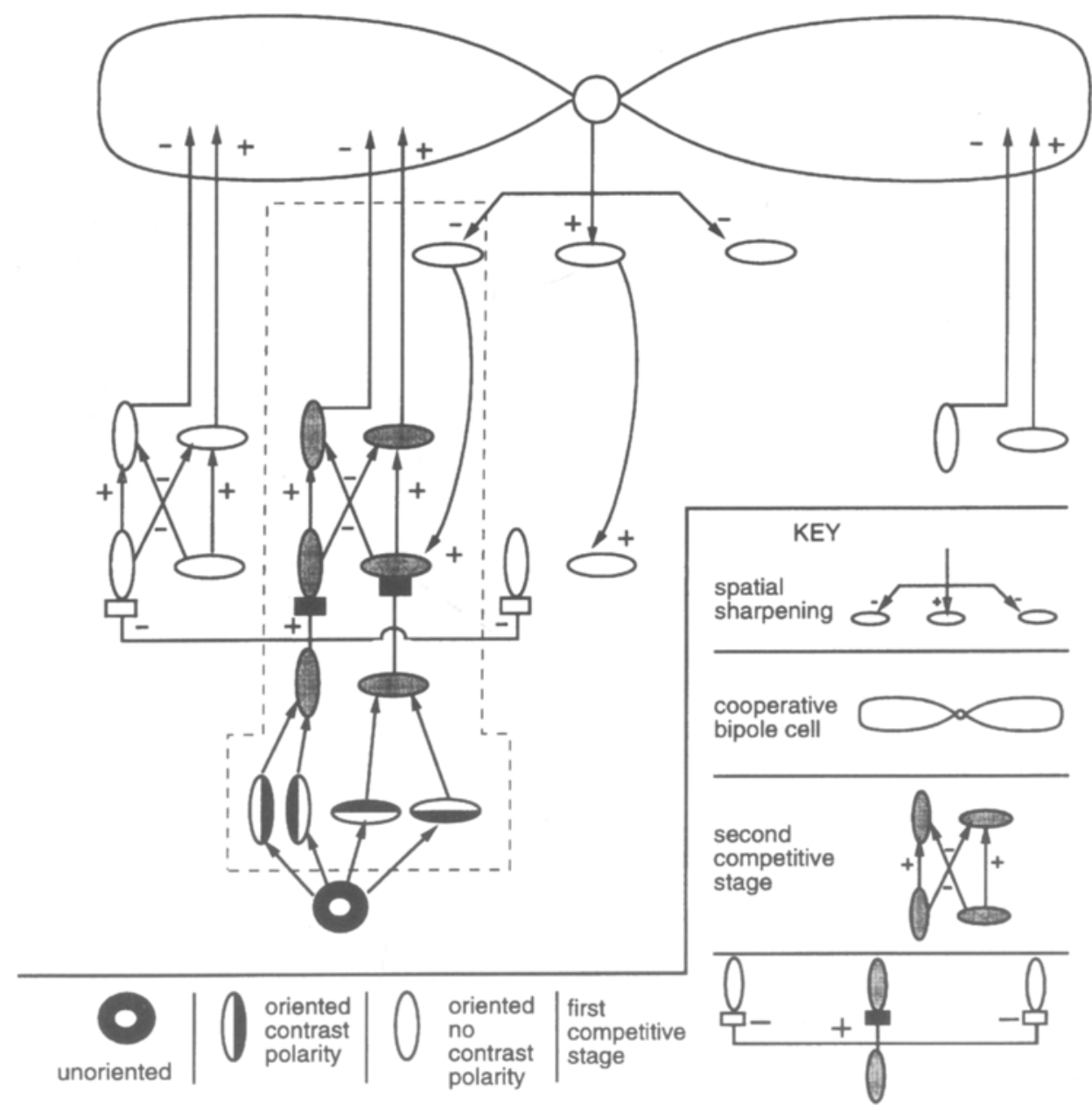

Figure 1. Schematic diagram of the boundary contour system. Cooperative and competitive interactions, embedded in a feedback neural network, process visual inputs. Reprinted, with permission, from Perception \& Psychophysics, 58, p.1 105. Copyright 1996 Psychonomic Society. 
stopping operation (Hubel \& Wiesel, 1965; Orban, Kato, $\&$ Bishop, 1979a, 1979b). These interactions are gated by habituative chemical transmitters. The hypercomplex cells input to a competition across orientation (second competitive stage) among higher order hypercomplex cells. Their outputs identify the location and orientation of stimulus edges or boundaries. They feed into cooperative bipole cells which, in turn, generate feedback signals that enhance spatially and orientationally consistent oriented patterns of activation while inhibiting (spatial sharpening) inconsistent ones. Like the cells reported to exist in area V2 (von der Heydt, Peterhans, \& Baumgartner, 1984), model bipole cells require substantial excitatory input on each half of their bow-tie-shaped receptive field. In response to visual input, feedback among BCS bipole cells and hypercomplex cells helps to drive a cooperative-competitive decision process that completes the statistically most favored patterns and suppresses less favored patterns of activation. Cooperating activations are said to resonate in the network.

Each cell in the BCS has its own local dynamics involving activation by inputs and passive decay (of the order of simulated milliseconds). However, excitatory feedback loops dominate the temporal properties of the BCS. When inputs feed into the BCS, they trigger reverberatory circuits that are not easily stopped. Simulations in Francis et al. (1994) demonstrate that, if left unchecked, these reverberations can last for hundreds of simulated milliseconds. However, the spatial structure of the bipole cells' receptive fields limits the persistence of resonating activities. A bipole cell requires excitatory inputs on both sides of its receptive field; thus, when the visual inputs disappear, cells centered on the middle of an image contour receive strong bipole feedback, but cells centered near the end receive no feedback. At stimulus offset, the activity of a cell centered at the end of an image contour passively decays away. This exposes a new cell at the contour end, which stops receiving bipole feedback and passively decays away as well. This erosion process continues from the contour ends to the middle of the contour, as described in Francis et al. (1994). As cells drop out of the feedback loop, the loop contains less activity, thereby weakening the feedback signals so that erosion accelerates. Eventually the feedback loop no longer contains enough activity to support itself and the resonance collapses.

Erosion occurs slowly relative to the duration of a brief stimulus and, if unchecked, could lead to undesirably long persistence after stimulus offset and thus smearing in response to motion. The BCS needs to use resonant feedback to complete patterns of activation for unmoving scenic objects, even as it actively resets the same activations when the objects change. Francis et al. (1994) identified two mechanisms embedded in the BCS design that reset resonant activations. One mechanism, called a gated dipole, produces reset signals at stimulus offset that actively inhibit the persisting activations. Habituation of chemical transmitters (boxes in Figure 1) shifts the balance of activity among competing pathways in the second compet- itive stage. At the offset of a stimulus, this shift creates rebounds of activity in nonstimulated pathways. Within the BCS, these rebounds inhibit the persisting activations. The properties of these reset signals explain why persistence of static stimuli varies inversely with stimulus duration and luminance (e.g., Bowen, Pola, \& Matin, 1974), why the persistence of illusory contours is greater, and differently affected by stimulus duration, than luminance contours (Meyer \& Ming, 1988), and how orientationspecific adaptation can increase or decrease persistence (Meyer, Lawson, \& Cohen, 1975). Details of these properties are in Francis et al. (1994).

A second source of resetting inhibition comes from the lateral inhibition of the first competitive stage. Because the model lateral inhibition strengthens with closer retinal separation, decreasing the spatial separation between successive stimuli decreases the persistence of the first stimulus. These results are consistent with the persistence data of Farrell, Pavel, \& Sperling (1990), among others. The next section demonstrates that the reset signals and lateral inhibition also account for properties of temporal integration.

\section{TEMPORAL INTEGRATION: SIMULATIONS}

The goal of this study is to identify neural network mechanisms that robustly explain the properties of visual persistence and temporal integration. As in Francis et al. (1994), the properties described here hold across a wide range of parameter choices because the model characteristics are due to the basic network design. Such robustness is highly desirable because it suggests that even as the BCS model evolves to fit other properties of visual perception, it will continue to explain the trends of persistence and temporal integration data. All simulations described in this paper use a single set of parameters.

Direct application of the intrinsic persistence hypothesis suggests that the likelihood of temporal integration increases with the duration of overlap among the signals generated by the two displays. The current simulations track the coexistence of the model signals generated at the second competitive stage (Level 6; see Appendix in Francis et al., 1994, for details). Longer durations of coexistence should correspond to better performance on the integration task.

It is premature to hypothesize the relationship between duration of coexistence and percentage correct on the integration task. To make the current simulations computationally feasible, the receptive fields of cells in the BCS model were "stripped down" relative to simulations that study the spatial characteristics of the BCS. Because of these simplifications, the spatial layouts of the simulated displays differ from those of the originals. In each simulation, the leading display consists of a single luminous bar. The second display consists of two bars that flank the first. The differences from the original displays and the simplifications necessary to make the simulations computationally feasible prevent the formation of a map- 
ping between coexistence and percentage correct. However, the direction and magnitude of change in duration of coexistence due to a change in display parameters do suggest a similar change in percentage correct. This approach will undoubtedly break down at very short or very long durations, where corresponding psychophysical studies will show floor or ceiling effects. Their influence will be noted when necessary. The following sections describe the properties of temporal integration and the model's explanation.

\section{Interstimulus Interval}

As the ISI between frames of the display increase, performance on the integration task drops. The effect of ISI is evident in Figure 2a (from Di Lollo et al., 1994), which shows results for 1 subject, where darker shading indicates poorer performance (percentage correct) on a temporal integration task. (The dashed and solid lines on the figure show predictions from various models considered by Di Lollo et al., 1994.) The changes in shading indicate the boundaries between $0 \%$ and $25 \%, 25 \%$ and $50 \%$, $50 \%$ and $75 \%$, and $75 \%$ and $100 \%$. For fixed display duration, increasing the ISI produces worse performance. Two other subjects showed similar characteristics.

Figure $2 b$ shows the relative durations of coexistence of activations generated by the two frames of the integration display. Darker shading indicates shorter coexistence and implies poorer performance on a corresponding integration task. The solid lines indicate equal-value contours. Looking up any column of Figure $2 \mathrm{~b}$ shows that duration of coexistence decreases as the ISI increases. This result follows naturally from the intrinsic persistence hypothesis. The activities generated by the first dis- play persist through the ISI and coexist with activities generated by the second display. As the ISI increases, less persisting activity remains to coexist with the second display.

\section{Inverse Duration With Leading Display}

Di Lollo et al. (1994) also replicated the inverseduration effect of temporal integration studies. Figure $2 a$ shows that increases in the duration of the elements of the first display result in poorer performance on the integration task. (The duration of the trailing display was fixed at $10 \mathrm{msec}$.)

In the context of the BCS model, this result too can be explained by the intrinsic persistence hypothesis. As described in Francis et al. (1994), offset of a luminance edge produces a rebound of activation in a competing pathway, which then inhibits the resonating activities. The strength of the rebound depends on the amount of habituation in depletable gates, which in turn depends on the duration of the luminance edge. Thus, increasing the duration of the elements of the first display results in stronger reset signals, shorter persistence of resonating activities, and poorer integration. Looking across any row of Figure $2 b$ shows that as display duration increases, the duration of coexistence of signals decreases.

\section{Inverse Intensity}

In a second experiment, Di Lollo et al. (1994) investigated how changes in stimulus intensity affected integration. In Figure $3 a$, the ISI necessary for 1 subject to achieve $50 \%$ correct on the integration task is plotted for various combinations of leading display duration and for dim or bright display elements. Figure 3 a shows that the critical
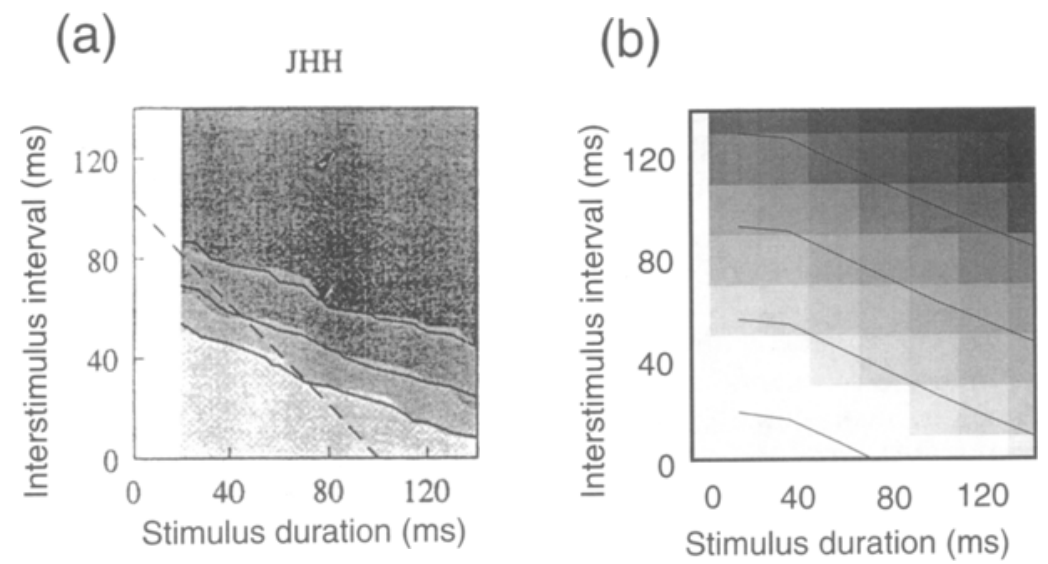

Figure 2. Increasing the duration of the leading display or the ISI hurts performance. (a) Psychophysical data. Gray-scale measure of percentage correct in a temporal integration task. Darker regions indicate poorer performance. The solid and dashed lines mark predictions of theories considered in the original study. (From "Temporal Integration and Segregation of Brief Visual Stimuli: Patterns of Correlation in Time," by V. Di Lollo, J. H. Hogben, and P. Dixon, 1994, Perception \& Psychophysics, 55, p. 377. Copyright 1994 by the Psychonomic Society, Inc. Reprinted with permission.) (b) Results of computer simulations. Gray-scale measure of coexistence of activities from the leading and trailing stimuli. Darker regions indicate shorter durations of coexistence. The solid lines mark equal value contours. 
(a)

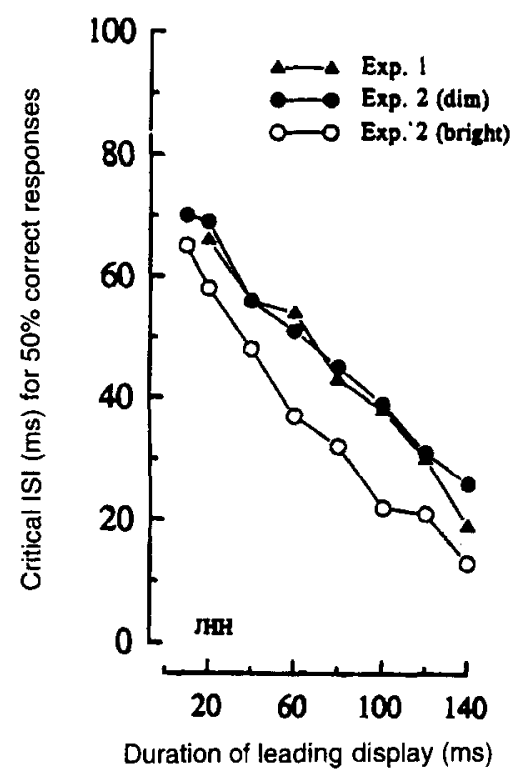

(b)

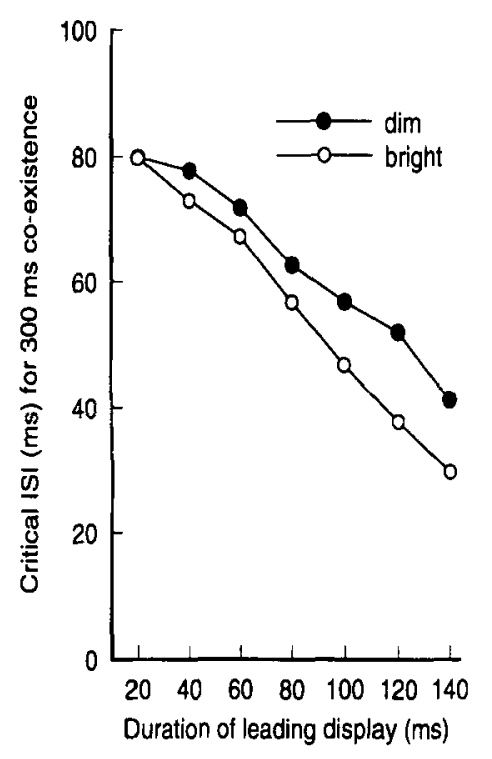

Figure 3. Increasing the intensity or the duration of the display elements hurts performance. (a) Psychophysical data. The critical ISI needed to achieve $50 \%$ correct on the integration task decreases as display luminance and duration increase. (From "Temporal Integration and Segregation of Brief Visual Stimuli: Patterns of Correlation in Time," by V. Di Lollo, J. H. Hogben, and P. Dixon, 1994, Perception \& Psychophysics, 55, p. 383. Copyright 1994 by the Psychonomic Society, Inc. Reprinted with permission.) (b) Results of computer simulations. The critical ISI needed to have coexistence of signals with a duration of $\mathbf{3 0 0}$ msec decreases as display luminance and duration increase.

ISI is smaller as display duration increases (due to the inverse-duration effect described above), and that increasing the intensity of the display elements results in shorter critical ISIs. This means that the task is more difficult for the brights dots than for the dim dots. Also plotted are the critical ISI values from the data in Figure 2a.

This result is consistent with the intrinsic persistence hypothesis in the BCS model. Two factors in the model contribute to the inverse intensity effect. First, the amount of habituation in the depletable gates depends on the strength of the signal passing through. Thus, more intense stimuli produce more habituation and stronger reset signals at stimulus offset. The stronger reset signals shorten the persistence of resonating activities in the network. Second, more intense stimuli in the trailing display produce stronger lateral inhibition (described below), which further speeds up the erosion process and reduces persistence of the leading display elements. As a result, the ISI necessary to reach a threshold level of coexistence in the integration task is smaller for bright than for dim stimuli. Figure $3 \mathrm{~b}$ plots critical ISI values for simulations using bright and dim display elements. The results qualitatively match the data except at very short display durations.

\section{Inverse Duration With Trailing Display}

Dixon and Di Lollo (1994) replicated findings from Groner et al. (1988) that increases in the duration of the trailing display results in poorer integration. Figure 4a shows percentage correct on the integration task as a function of trailing display duration, with separate curves for different leading display durations. The ISI between displays was zero. The data replicate the inverse duration effect of the leading display discussed above. The data also show that similar changes in performance occur as the duration of the trailing display increases. Both of these effects exist in displays with bright and dim illumination, although there are quantitative differences between these conditions.

Again, in the context of the BCS model, these results can be explained by considering the persistence of leading display elements. In the BCS model, lateral inhibition from elements of the trailing display shortens the persistence of activities generated by the elements of the first display. Increasing the duration of the trailing display generates longer lasting inhibition, which results in shorter persistence. As above, with the intrinsic persistence hypothesis, shorter persistence of activities generated by the leading display results in poorer performance on the integration task. Figure $4 \mathrm{~b}$ plots the duration of network activity coexistence as a function of trailing display duration, for various leading display durations. The results qualitatively agree with much of the data.

The model does fail to match the effects produced by changing illumination. The model produces an inverse contrast effect. (The elements are more luminous, relative 

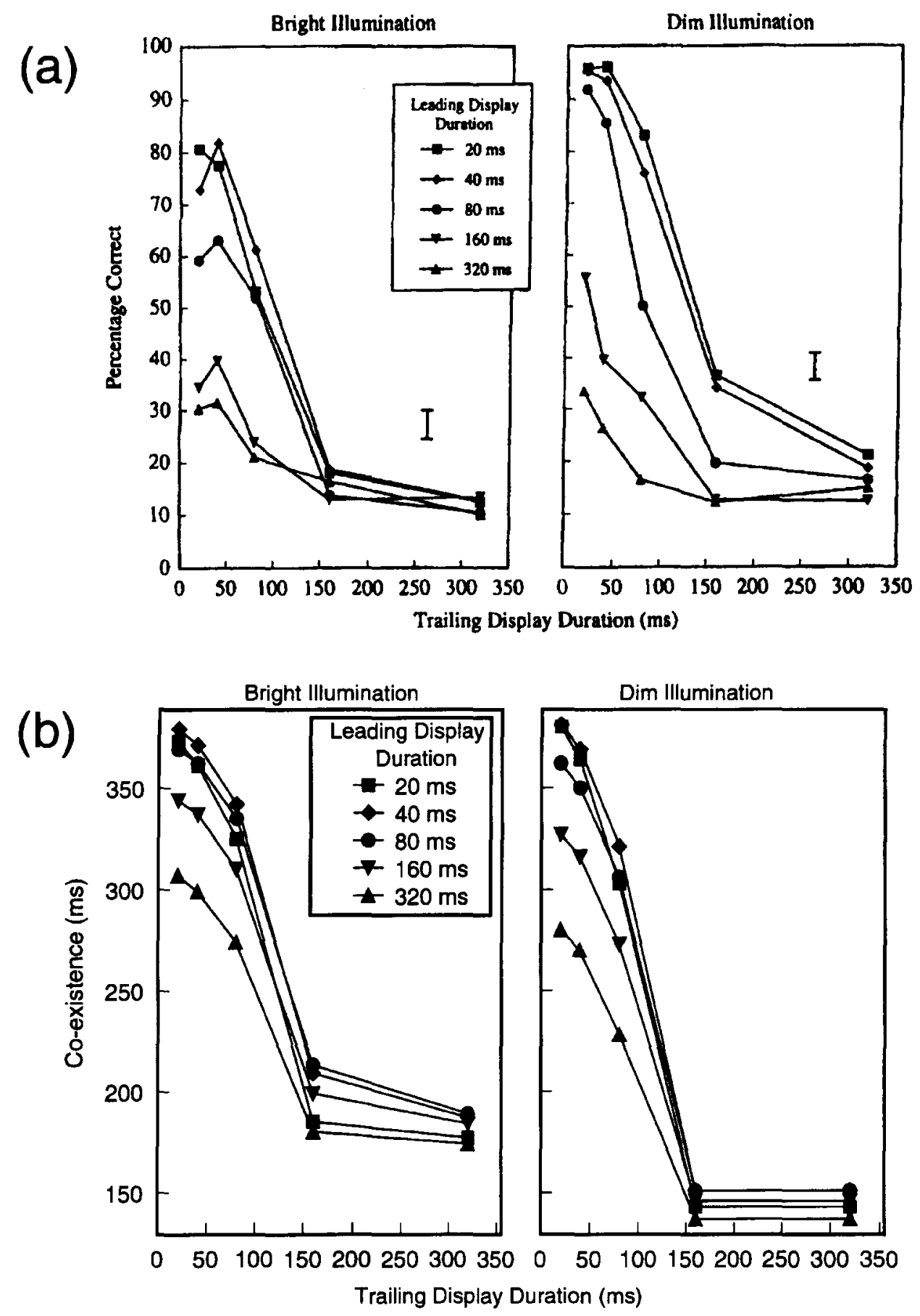

Figure 4. Increasing the duration of the leading or trailing display hurts performance. (a) Psychophysical data. (From "Beyond Visible Persistence: An Alternative Account of Temporal Integration and Segregation in Visual Processing," by P. Dixon and V. Di Lollo, 1994, Cognitive Psychology, 26, pp. 33-63. Copyright 1994 by Academic Press. Reprinted with permission.) (b) Results of computer simulations. Duration of coexistence is assumed to be related to percentage correct, where increased coexistence in the model's response is related to an increase in discrimination.

to the background, in the dim-illumination condition than in the bright-illumination condition.) As a result, coexistence duration is shorter with dim illumination than with bright illumination. A number of psychophysical studies show that increasing the background luminance leads to shorter persistence (see the review by Di Lollo \& Bischof, 1995). The model is unable to account for these findings when increases in background luminance do not also lead to increases in contrast. This may be because background luminance affects unmodeled retinal processes. 


\section{Inverse Proximity}

An inverse proximity effect provides additional evidence that lateral inhibition affects temporal integration. Each curve in Figure 5a (from Di Lollo \& Hogben, 1987) plots the percentage of errors in an integration task as a function of stimulus onset asynchrony (SOA) for various spatial separations of the display elements. (Since the stimuli had fixed durations, variations in SOA were the same as variations in ISI.) The data show that increasing the spatial separation between display elements results in fewer errors.

The model explains these results using lateral inhibition. Through the first competitive stage, the trailing display's elements inhibit the persistence of the leading display's elements. As above, with the intrinsic persistence hypothesis, shorter persistence of the leading display's elements leads to poorer integration. Figure 5 b plots the simulated duration of activity coexistence as a function of SOA for various spatial separations. (The $y$-axis in Figure $5 \mathrm{~b}$ is inverted, because shorter durations of boundary coexistence should result in more errors.)

There are differences between the psychophysical data and simulated results. The simulated results show evidence of lateral inhibition even at the shortest SOA, while the data reveal such interactions only at longer SOAs. One explanation of this effect is that there is a ceiling effect for coexistence of signals beyond 355 simulated milliseconds, where performance on the integration task would be effectively errorless. In Figure $5 \mathrm{~b}$, this would suggest that any point below the line $y=355$ would lie on a common horizontal line when plotted as percentages of er- rors. As can be checked by hand, the resulting figure looks more similar to the data curves.

A second explanation is that the lateral inhibition may be the same type as described in metacontrast masking studies, and reveals itself only at intermediate SOAs. Di Lollo and Hogben (1987) proposed this explanation and suggested that transient on sustained inhibition, described by Breitmeyer and Ganz (1976), might explain their findings. In this theory, maximal inhibition occurs at intermediate SOAs because of differences in response lags between transient and sustained cells. The current simulations do not include these characteristics.

\section{PREDICTIONS}

The model provides predictions of performance on two temporal integration displays. The predictions depend on the properties of lateral inhibition in the model. It must be emphasized that the simulations predict general trends; they do not predict the display parameters necessary to produce these results. For example, the spatial range of lateral inhibition in the current simulations extends to $21 \mathrm{~min}$, which probably underestimates the range of inhibition in vivo. Additionally, the current simulations do not include an accurate model of retinal processing, so any predictions about luminance effects must be qualitative rather than quantitative.

\section{Inverse Proximity and Trailing-Display Duration}

The BCS model's explanation of the data in Figure 4a, which shows that integration performance decreases as
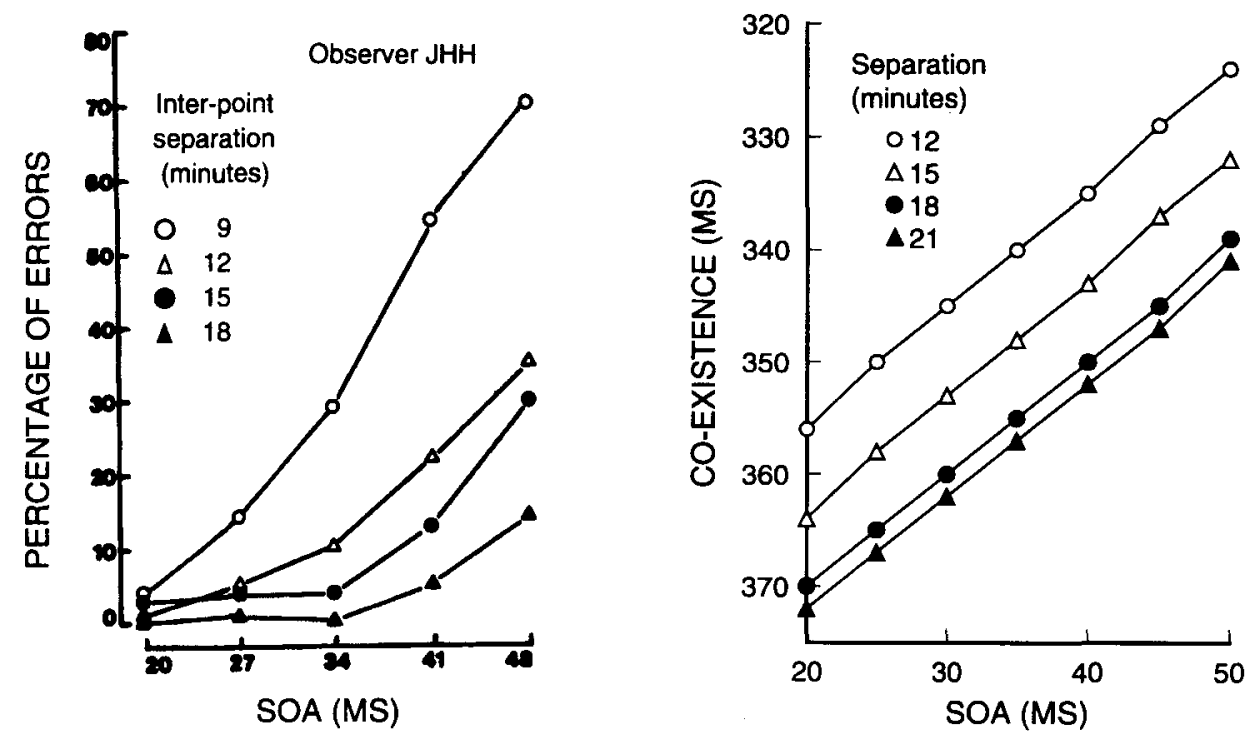

Figure 5. Decreasing spatial separation hurts performance. (a) Psychophysical data. (From "Suppression of Visible Persistence as a Function of Spatial Separation Between Inducing Stimuli," by V. Di Lollo and J. H. Hogben, 1987, Perception \& Psychophysics, 41, p. 352. Copyright 1987 by the Psychonomic Society, Inc. Reprinted with permission.) (b) Results of computer simulations. Shorter durations of coexistence are assumed to be related to higher error percentages. 
trailing display duration increases, depends on inhibitory signals sent from the elements of the second display to persisting representations generated by the elements of the first display. This is quite different from the explanation of the data by Dixon and Di Lollo (1994). Using the temporal coding hypothesis, they suggested that the decreased performance was due to the corresponding decrease in correlation between responses generated by such displays. A key difference between these explanations is the role of spatial separation. The model proposed by Dixon and Di Lollo should show no variation in performance as a function of spatial separation, while the BCS model suggests that the effects of trailing display duration should weaken with spatial separation.

Figure 6a plots the duration of coexistence of model signals as a function of trailing display duration for various spatial separations. The model predicts that increasing the separation between elements decreases the effect of the duration of the trailing display. The model further predicts that the difference in separation should be strongest for long trailing-display durations.

\section{Inverse Proximity and Trailing-Display Intensity}

The model further predicts that changes in intensity of the trailing display should have qualitatively similar effects as changes in duration of the trailing display. While increasing the duration of the trailing display produces longer lasting inhibition, increasing the luminance should produce stronger inhibition. In each case, the stronger net inhibition should reduce the persistence of the leading display elements and hurt performance on the integration task.

Moreover, because the strength of lateral inhibition is modulated by spatial separation, the effect of luminance changes should be weakest for large spatial separations. Figure $6 \mathrm{~b}$ plots the duration of the coexistence of model signals as a function of trailing-display intensity for various spatial separations. The model predicts that as spatial separation increases, the inverse intensity with trailingdisplay effect weakens.

\section{DISCUSSION}

The simulations described in this paper show that the intrinsic persistence hypothesis can explain properties of temporal integration if it is applied to the BCS model. More significantly, the model provides a unified explanation of temporal integration data that have previously been accounted for only in piece-meal fashion with theories of limited applicability. For example, alternative explanations of the inverse-proximity effect include several versions of lateral inhibition (Castet, 1994; Di Lollo \& Hogben, 1987; Farrell et al., 1990), but all fail to explain other properties of integration such as the inverseduration effects and the inverse-intensity effects. In terms of data coverage, the most comprehensive alternative to the BCS model is the temporal coding model proposed by Dixon and Di Lollo (1994), which accounts for all the (a)

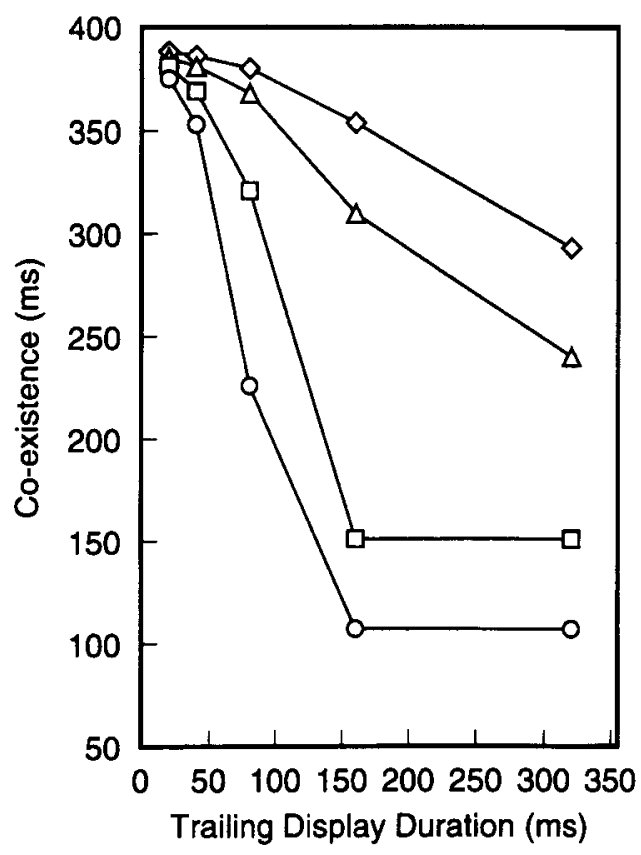

(b)

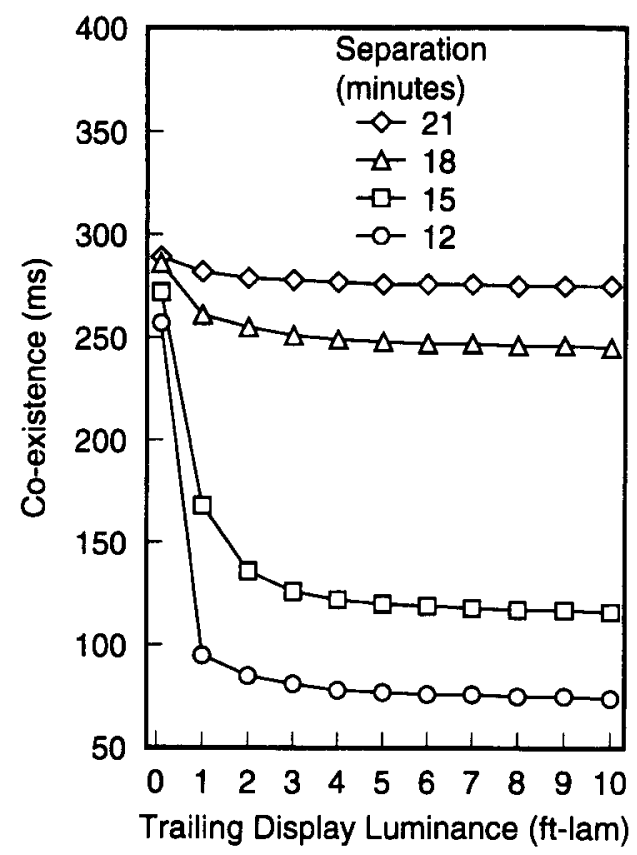

Figure 6. Predictions of the model. (a) The effect of changing the duration of the trailing display weakens as spatial separation increases. (b) The effect of changing the luminance of the trailing display weakens as spatial separation increases. 
data discussed here except the inverse-proximity effect. (The model proposed by Groner et al., 1988, is mathematically similar and could probably account for the same data sets.) In contrast to these limited models, the BCS provides a unified explanation of all these data with the same mechanisms used to explain properties of visual persistence.

The explanatory scope of the BCS model extends much further, as it links both persistence and integration data to mechanisms necessary for spatial processing. The feedback and inhibitory mechanisms used here to explain characteristics of dynamic perception also play important roles in spatial perception. Thus, the BCS model is unifying diverse psychophysical data on dynamic vision with mechanisms that are also consistent with a large set of spatial characteristics. The dynamic emergent properties used to explain temporal integration are consistent with, and depend upon, the BCS's roles in boundary completion, texture segregation, shape from shading, brightness perception, 3-D vision, and motion processing, among others.

\section{REFERENCES}

Bowen, R., POLA, J., \& MATIN, L. (1974). Visual persistence: Effects of flash luminance, duration and energy. Vision Research, 14, 295-303.

BREITMEYER, B., \& GANZ, L. (1976). Implications of sustained and transient channels for theories of visual pattern masking, saccadic suppression, and information processing. Psychological Review, 83, 1-36.

BURR, D. (1980). Motion smear. Nature, 284, 164-165.

CASTET, E. (1994). Effect of the ISI on the visible persistence of a stimulus in apparent motion. Vision Research, 34, 2103-2114.

Di Lollo, V., \& BischoF, W. (1995). Inverse-intensity effect in duration of visible persistence. Psychological Bulletin, 118, 223-237.

Di Lollo, V., \& Hogben, J. H. (1987). Suppression of visible persistence as a function of spatial separation between inducing stimuli. Perception \& Psychophysics, 41, 345-354.

Di Lollo, V., HogBeN, J. H., \& Dixon, P. (1994). Temporal integration and segregation of brief visual stimuli: Patterns of correlation in time. Perception \& Psychophysics, 55, 373-386.

Dixon, P., \& Di Lollo, V. (1994). Beyond visible persistence: An alternative account of temporal integration and segregation in visual processing. Cognitive Psychology, 26, 33-63.

Farrell, J., Pavel, M., \& SPerling, G. (1990). The visible persistence of stimuli in stroboscopic motion. Vision Research, 30, 921-936.

Francis, G., Grossberg, S., \& Mingolla, E. (1994). Cortical dynamics of feature binding and reset: Control of visual persistence. Vision Research, 34, 1089-1104

Groner, M., Bischof, W., \& Di Lollo, V. (1988). A model of visible persistence and temporal integration. Spatial Vision, 3, 293-304.

GrossBERG, S. (1994). 3-D vision and figure-ground separation by visual cortex. Perception \& Psychophysics, 55, 48-120.

Grossberg, S., \& Mingolla, E. (1985a). Neural dynamics of form perception: Boundary completion, illusory figures, and neon color spreading. Psychological Review, 92, 173-211.

Grossberg, S., \& Mingolla, E. (1985b). Neural dynamics of perceptual grouping: Textures, boundaries, and emergent segmentations. Perception \& Psychophysics, 38, 141-171.

HABER, R. (1983). The impending demise of the icon: A critique of the concept of iconic storage in visual information processing. Behavioral \& Brain Sciences, 6, 1-54.

Hogben, J. H., \& Di Lollo, V. (1974). Perceptual integration and perceptual segregation of brief visual stimuli. Vision Research, 14, 10591069.

HUBEL, D., \& Wiesel, T. (1965). Receptive fields and functional architecture in two nonstriate visual areas (18 and 19) of the cat. Journal of Neurophysiology, 28, 229-289.
LUdTKE, D. (1992). NXPlot3d (Version 3.0) [Computer software]. Houston, TX: Rice University, Department of Physics (web address: http://ion.rice.edu/nsci/plotting/nxplot3d.html).

MEyer, G., LAWSON, R., \& COHEN, W. (1975). The effects of orientationspecific adaptation on the duration of short-term visual storage. $\mathrm{Vi}$ sion Research, 15, 569-572.

MeYer, G., \& Ming, C. (1988). The visible persistence of illusory contours. Canadian Journal of Psychology, 42, 479-488.

Orban, G., Kato, H., \& Bishop, P. (1979a). Dimensions and properties of end-zone inhibitory areas in receptive fields of hypercomplex cells in cat striate cortex. Journal of Neurophysiology, 42, 833-849.

Orban, G., Kato, H., \& Bishop, P. (1979b). End-zone region in receptive fields of hyper-complex and other striate neurons in the cat. Journal of Neurophysiology, 42, 818-832.

von der Heydt, R., Peterhans, E., \& Baumgartner, G. (1984). Illusory contours and cortical neuron responses. Science, 224, 1260-1262.

\section{APPENDIX}

\section{Simulation}

The network equations are identical to those used in Francis et al. (1994). Readers interested in the equations are referred to that manuscript. The current simulations make only three changes from those earlier simulations. First, the parameter $K=0.00003$, which controls the rate of habituation in the gated dipole circuits, is substantially smaller than in Francis et al. (1994). This change was necessary to achieve a better quantitative fit with the data in Figure 2. Second, the kernels of Level 1 center-surround cells were restricted to include only the central pixel. This change was necessary because larger kernels created extraneous boundary signals (which were not created with the different stimuli investigated in Francis et al., 1994). Third, to save computation, the BCS simulations included only horizontal cells (the orientation of the stimuli). These changes do not affect the analysis of persistence data described in Francis et al. (1994), although they may affect the quantitative fits.

All computations were on a $30 \times 40$ pixel grid. As in Francis et al. (1994), symmetry arguments required computation of only one quadrant of the image plane, thereby reducing the computation to a $15 \times 20$ grid. All equations were solved using Euler's method with a step size of 0.01 . Duration of boundary coexistence for all the simulations was measured by noting the time when any Level 6 signals of the stimuli were both above a constant threshold of 0.5 . Activities of cells were checked every 0.1 time step ( 1 simulated millisecond) to measure boundary signal coexistence. To illustrate computation time, each curve in Figure $4 \mathrm{~b}$ takes approximately $4 \mathrm{~h}$ to calculate on a dedicated IBM RS6000 355 POWERstation.

\section{Stimuli}

For each simulation, the first display contained a bright bar, 1 pixel wide and 30 pixels long, centered on the simulation display. The second display consisted of a pair of flanking bars. The display during the ISI was always the background luminance.

For the simulations summarized in Figure $2 b$, the trailing display was always shown for 20 simulated milliseconds. Di Lollo et al. (1994) used a brightness equalization procedure, but do not give the resulting luminance values of the stimuli. The simulations use luminance values given in Dixon and Di Lollo (1994), who used the same brightness equalization procedure. As a result, $20-\mathrm{msec}$ stimuli were displayed at $120 \mathrm{~cd} / \mathrm{m}^{2}, 40-\mathrm{msec} \mathrm{stim}-$ uli at $60 \mathrm{~cd} / \mathrm{m}^{2}, 60-\mathrm{msec}$ stimuli at $49.5 \mathrm{~cd} / \mathrm{m}^{2}, 80-\mathrm{msec}$ stimuli at $39 \mathrm{~cd} / \mathrm{m}^{2}$, and $100-, 120-$, and $140-\mathrm{msec}$ stimuli at $35 \mathrm{~cd} / \mathrm{m}^{2}$. Background luminance was $0.1 \mathrm{~cd} / \mathrm{m}^{2}$. All these values were converted to footlamberts for the simulations. The elements of 
the first and second displays were separated by 7 pixels. The longest duration of coexistence in Figure $2 \mathrm{~b}$ was 390 simulated milliseconds, the shortest duration of coexistence was $196 \mathrm{sim}$ ulated milliseconds. The density plot in Figure $2 \mathrm{~b}$ was made with the program NXPlot3d (Ludtke, 1992).

The simulations summarized in Figure $3 \mathrm{~b}$ were the same as in Figure 2b, except that each display element was $1 / 10$ as luminous (dim condition) or 10 times as luminous (bright condition). The critical ISI value for each display duration was calculated from a linear regression fit to the points generated by fixing display duration and varying ISI.

For the simulations summarized in Figure $4 b$, the 160 - and $320-\mathrm{msec}$ displays had luminances of $35 \mathrm{~cd} / \mathrm{m}^{2}$. Background luminance was $0.1 \mathrm{~cd} / \mathrm{m}^{2}$ in the dim condition and $10.0 \mathrm{~cd} / \mathrm{m}^{2}$ in the bright condition. The elements of the first and second displays were separated by 5 pixels.

For the simulations summarized in Figure 5 b, both displays were presented for 10 simulated milliseconds at $103 \mathrm{~cd} / \mathrm{m}^{2}$ on a dark $\left(0.1 \mathrm{~cd} / \mathrm{m}^{2}\right)$ background. The second display elements were presented 4 to 7 pixel spaces from the first display elements. As in Francis et al. (1994), each pixel space corresponds to 0.05 visual degrees.

The simulations summarized in Figure $6 \mathrm{a}$ were the same as for Figure $4 \mathrm{~b}$, except that the spatial separation varied between 4 and 7 pixels. The leading display duration was fixed at 40 simulated milliseconds, and the background luminance was set to $0.1 \mathrm{~cd} / \mathrm{m}^{2}$.

The simulations summarized in Figure $6 \mathrm{~b}$ used display durations of 100 simulated milliseconds and an ISI of 60 simulated milliseconds. The luminance of the leading display was $5 \mathrm{fL}$. The smallest luminance of the trailing display was $0.1 \mathrm{fL}$, and then varied in unit steps from 1 to $10 \mathrm{fL}$. The background luminance was $0.0292 \mathrm{fL}$.

(Manuscript received February 10, 1995; revision accepted for publication February 5, 1996.) 